\title{
Comparison of Detection of Giardia lamblia and Entamoeba histolytica Using Microscopy and Antigen Detection Rapid Cards in Patients with Diarrhea
}

\author{
Sarumathi Dandapani ${ }^{1}$, Nonika Rajkumari² ${ }^{2}$, Pallam Gopichand ${ }^{3}$
}

\begin{abstract}
Background and objectives: Intestinal infections by protozoan parasites like Giardia lamblia and Entamoeba histolytica is still much prevalent, more so in developing countries. Microscopy remains the mainstay of its diagnosis despite its limitations. Therefore, we have tried to assess the usefulness of using antigen based rapid card test to that of the routine stool microscopy in this study and see its associated risk factors.

Materials and methods: Stool samples were collected from enrolled patients with history of loose motion. On these, routine stool microscopy was followed by a rapid combo test for antigen detection of Entamoeba, Giardia and Cryptosporidium. The associated risk factors of these patients were then checked and analyzed.

Results: A total of 2,434 stool samples were included in the study without duplication, of which 790 samples were found positive for an intestinal parasite by microscopy and 249 were positive by the rapid triage card test for one of the three intestinal protozoan parasites. Both cysts and trophozoites of G. Iamblia and Entamoeba spp. were recovered from these samples. Stool microscopy for Giardia was positive in 17 samples and 60 for Entamoeba spp. However, by rapid card test, 114 samples were found to be positive for G. lamblia, 129 for Entamoeba spp. and 51 for Cryptosporidium spp. The additional detection of these intestinal pathogen by the rapid cards were found to be statistically significant $(p<0.005)$. Conclusions: The results show that routine screening for intestinal parasitosis is important as many persons can have silent infection. In addition, a combination of another test especially rapid test along with microscopy helps to identify such cases.
\end{abstract}

Keywords: Diarrhea, Entamoeba histolytica, Giardiasis, Malabsorption, Rapid test.

Journal of Gastrointestinal Infections (2020): 10.5005/jp-journals-10068-3036

\section{INTRODUCTION}

Intestinal parasitic infections are one of the major causes of morbidity and mortality worldwide. Amebiasis, giardiasis, hookworm infection, ascariasis, and trichuriasis are the most common parasitic infections seen among the community. They are responsible for iron-deficiency anemia, chronic diarrhea, portal hypertension and malnourishment in children along with other comorbidities. ${ }^{1}$ Giardiasis caused by Giardia duodenalis (otherwise, G. intestinalis, G. lamblia), is one of the established intestinal protozoan flagellate of the humans..$^{2-4}$ Giardiasis is widespread globally and it is common in both children and adults. The prevalence of Giardia infection is more in underdeveloped and developing countries. Poor socioeconomic conditions such as inadequate sanitation, poor hygienic practices and illiteracy are identified risk factors for intestinal parasitic diseases in the communities. ${ }^{5}$ World Health Organization has included giardiasis in the "neglected diseases initiative". ${ }^{6}$ The life cycle of Giardia species involves two forms of the parasite such as trophozoite and cystic forms. Giardia transmission occurs through the ingestion of the infective cyst stage shed in human or animal feces. ${ }^{7}$ The cyst has potential to remain infectious for several weeks. ${ }^{3}$ Another important protozoan parasite also follows a similar pattern but causes invasive diarrhea is Entamoeba histolytica.

The mode of transmission of both is via fecal-oral route by ingestion of cysts. After ingestion of cysts, the incubation period is approximately $9-15$ days. $^{8}$ The clinical manifestations for giardiasis vary from asymptomatic to acute watery, foul smelling diarrhea, nausea, epigastric pain and weight loss. ${ }^{8}$ Although many acute infections may cure spontaneously, it may also lead to the development of subacute or chronic infections. ${ }^{9}$ E. histolytica usually
${ }^{1-3}$ Department of Microbiology, Jawaharlal Institute of Postgraduate Medical Education and Research, Puducherry, India

Corresponding Author: Nonika Rajkumari, Department of Microbiology, Jawaharlal Institute of Postgraduate Medical Education and Research, Puducherry, India, e-mail: nonika.raj@gmail.com

How to cite this article: Dandapani S, Rajkumari N, Gopichand P. Comparison of Detection of Giardia lamblia and Entamoeba histolytica Using Microscopy and Antigen Detection Rapid Cards in Patients with Diarrhea. J Gastrointest Infect 2020;10(1):3-6.

Source of support: Nil

Conflict of interest: None

presents with an invasive disease mostly in the large intestine as amebic dysentery or if it spreads, as abscesses in liver, lungs or rarely in the brain.

Giardiasis as well as amebiasis can be identified by a simple stool microscopic examination. However, no single test or combination of two or more methods can detect all infections. To maximize the diagnostic sensitivity, three stool samples collected on alternate days is recommended due to intermittent shedding of G. lamblia and E. histolytica in stool. ${ }^{10}$ However, continued negative stool examination do not rule out both these infections. In addition, microscopy is not able to differentiate between the trophozoites/ cysts of E. histolytica/E. dispar/E. moshkovskii. The purpose of this study is to evaluate the distribution of symptomatic as well as asymptomatic giardia and entamoeba infections and factors associated with it.

(c) Jaypee Brothers Medical Publishers. 2021 Open Access This article is distributed under the terms of the Creative Commons Attribution 4.0 International License (https://creativecommons.org/licenses/by-nc/4.0/), which permits unrestricted use, distribution, and non-commercial reproduction in any medium, provided you give appropriate credit to the original author(s) and the source, provide a link to the Creative Commons license, and indicate if changes were made. The Creative Commons Public Domain Dedication waiver (http://creativecommons.org/publicdomain/zero/1.0/) applies to the data made available in this article, unless otherwise stated. 


\section{Materials and Methods}

\section{Setting and Study Design}

It is an observational study conducted in a tertiary care hospital in south India over a period of 4 years.

\section{Inclusion Criteria}

A cohort study carried out in a tertiary care hospital in south India from January 2016 to December 2019. Stool samples collected for suspected intestinal parasitic infections with loose motion from various departments like medicine, surgery, pediatrics, pulmonary medicine, gynecology, were examined microscopically by wet mount examination using direct saline and iodine mounts. They were also stained with permanent stool stains like trichrome and examined. Formal ether sedimentation concentration technique was also performed on the samples to check for any missed out intestinal parasitic cysts and eggs and examined microscopically. After this procedure, antigen detection triage cards (CerTest Crypto + Giardia + Entamoeba combo card, CerTest Biotech, Zaragoza, Spain) were used to test all the stool samples. This rapid card can detect E. histolytica, G. lamblia and Cryptosporidium spp. at one-step in the stool sample. Other species like E. dispar/E. moshkovskii believed to have controversial role in causing amebiasis can only be confirmed by polymerase chain reaction (PCR). Therefore, those samples coming positive for Entamoeba spp. by the rapid card test were confirmed by PCR for the species.

\section{Exclusion Criteria}

Only one stool sample from each patient was taken. Repeated stool samples from which Giardia and Entamoeba were already identified was excluded from the study during this period.

All results were calculated as means or percentages. A value of $p<0.005$ was taken as significant.

\section{Results}

The total number of stool samples received in the Microbiology laboratory was 2,434 . Total of 790 samples were found positive for an intestinal parasite by microscopy and 249 were positive by the rapid triage card test for one of the three intestinal protozoan parasites (Table 1). Of the total samples positive, stool microscopy for Giardia was positive in 17 samples. All the positive samples have cyst forms with three samples having trophozoite forms as well (Fig. 1). Among the positive cases, six cases were from pediatric age group. Fourteen out of 17 positive cases were from rural areas. Most of the patients were from medical gastroenterology department. Among these positive patients, three were symptomatic and detected while doing the routine investigations for other health issues.

Microscopy for Entamoeba spp was positive in 60 cases during the study period. All the positive samples have cyst forms among which five samples have trophozoite forms as well. Forty-five out of 60 positive cases were from rural areas. Most of the patients (71\%) were from medicine ward admitted with complaints of loose stools sometimes mixed with white mucus, whereas the remaining were distributed among the other departments which we have included in our study. Of these 60 positive samples with Entamoeba infection, one sample was found to have Entamoeba spp. and Giardia sp. coinfection. All the positive cases were identified by direct wet mount preparation of stool samples by saline and iodine mount followed by trichrome staining. Besides these two protozoan parasites, other intestinal parasites which were recovered include eggs of Ascaris lumbricoides, hookworm, Enterobius vermicularis, larvae of Strongyloides stercoralis, oocysts of Cryptosporidium spp., Cystoisospora belli etc. by microscopy. The distribution of positivity for these two parasites seen year wise is given in Table 2 .

However, by rapid card test, a total of 114 samples were found to be positive for G. lamblia whereas 129 were found positive for Entamoeba spp. and 51 for Cryptosporidium spp. (Table 1 and Fig. 2). All samples positive for microscopy were rapid card positive. These were found to be statistically significant $(p<0.005)$. These 129 samples positive for Entamoeba spp. were confirmed using our laboratory standardized protocol of nested multiplex PCR for E. histolytica/E. dispar/E. moshkovskii. ${ }^{11}$ It was seen that of the total 129 samples positive by rapid card test, 87 of them were confirmed as E. histolytica, 31 as

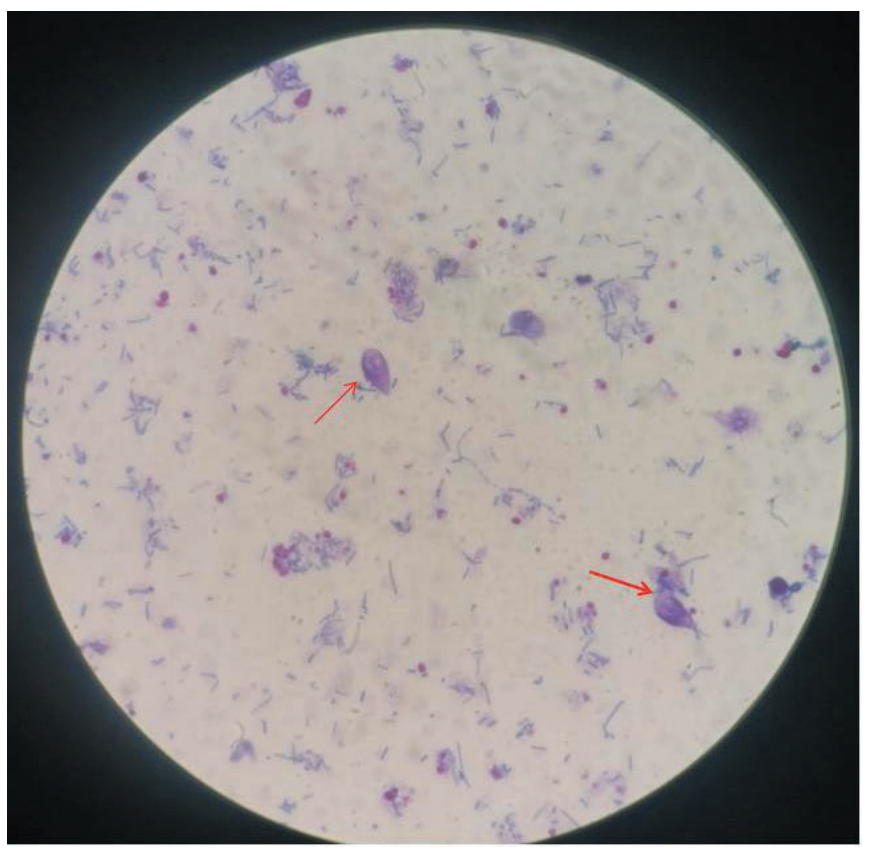

Fig. 1: Giardia lamblia trophozoites in trichrome stain smear

Table 1: Positivity of the intestinal parasites by microscopy and triage card test

\begin{tabular}{|c|c|c|c|c|c|c|c|}
\hline \multirow[b]{2}{*}{ Year } & \multirow[b]{2}{*}{ Total samples } & \multirow[b]{2}{*}{ Total positives } & \multicolumn{2}{|c|}{ Positive for Giardia } & \multicolumn{2}{|c|}{ Positive for Entamoeba } & \multirow[t]{2}{*}{ Others } \\
\hline & & & Microscopy & Rapid card & Microscopy & Rapid card & \\
\hline 2016 & 686 & 181 & 3 & 25 & 18 & 27 & 108 \\
\hline 2017 & 651 & 242 & 4 & 51 & 31 & 46 & 110 \\
\hline 2018 & 622 & 197 & 7 & 26 & 10 & 41 & 113 \\
\hline 2019 & 475 & 170 & 3 & 12 & 1 & 15 & 139 \\
\hline Total & 2434 & 790 & 17 & 114 & 60 & 129 & 490 \\
\hline
\end{tabular}


Table 2: Yearwise distribution of Giardia lamblia and Entamoeba spp. positive samples by microscopy

\begin{tabular}{llll}
\hline Year & Total number of stool samples & Giardia spp. & Entamoeba spp. \\
\hline 2016 & 686 & 3 & 18 \\
2017 & 651 & 4 & 31 \\
2018 & 622 & 7 & 10 \\
2019 & 475 & 3 & 1 \\
\hline
\end{tabular}
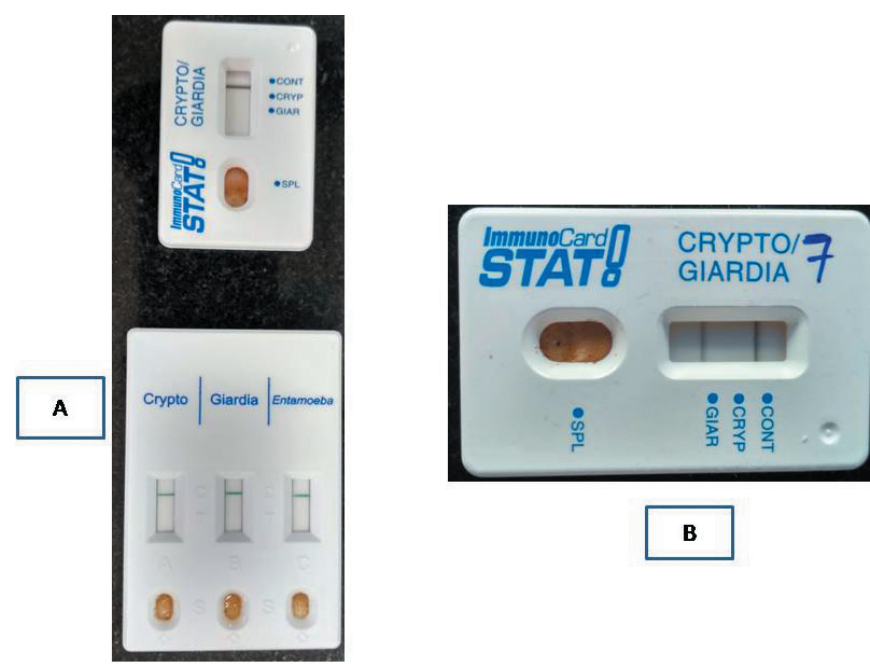

Fig. 2: Detection of the three intestinal parasites using rapid test (A) shows the negative test; (B) shows a sample testing positive for Giardia lamblia antigen

E. dispar and 11 as E. moshkovskii. This is also an important finding, which creates a big impact on the further management of the patient.

It was seen that associated comorbidities like irritable bowel syndrome, malabsorption, and growth stunting were seen in nine patients among those whose stool sample were positive for G. lamblia. Similar observation was also seen with those positive for Entamoebaspp. by microscopy but were not found to be statistically significant $(p=0.016)$.

\section{Discussion}

This study aimed to estimate the frequency, epidemiology and associated factors of Giardia and Entamoeba infections. We performed a stool survey in a tertiary care hospital in south India from January 2016 to December 2019. We aimed at procuring the socio-demographic data from these patients. The identification of Giardia spp. in stool samples by microscopy is the gold standard method for the diagnosis of giardiasis. ${ }^{12}$ Stool examination, because of its simplicity, has an important place in the diagnosis of giardiasis, especially in regions with high infection rates and limited diagnostic facilities. ${ }^{13}$ In this study; fecal samples were processed using floatation and sedimentation methods. The overall positivity of the infection by microscopy was $0.7 \%$ during the study period. However, a total of 114 were positive for Giardia antigen by rapid triage cards. These were seen even in patients who were asymptomatic. This is a significant finding in our study as this infection may be seen among the asymptomatic too.

In the vulnerable target group of children under five years of age, G. lamblia is the third most common agent of diarrheal disease. ${ }^{14}$ Infection with Giardia lamblia has also been linked to the development of irritable bowel syndrome and chronic fatigue..$^{15}$ The prevalence of giardiasis in humans ranges from 2 to $3 \%$ in industrialized countries, up to $30 \%$ in low-income and developing countries. ${ }^{16}$

People living in rural or under developed areas are more prone to the ingestion of infective form of parasites as compared to those who live in urban/suburban or well developed areas. ${ }^{17}$ A study by Fantinatti et al. ${ }^{18}$ attributed the high prevalence of giardiasis in study population to multiple factors such as poor sanitation and occurrence of anthropo-zoonotic transmission cycles. In our study, $83 \%$ of the patients with Giardia infection are from rural areas. In a study by Quihui-Cota et al. ${ }^{19} 23 \%$ of giardiasis was prevalent in pediatric age group. Also, in a study by Mukherjee et al. ${ }^{20}$ no significant association was found with seasonality which is similar to our study.

In case of E. histolytica infection, overall positivity of infection was $3 \%$ by microscopy during the study period, which is lesser than other parts of country. Studies from different geographical areas of the world reported that intensity of intestinal infection including $E$. histolytica was significantly higher among children, ${ }^{21,22}$ which is in contrast to our findings where majority of positive cases were from adult patients. Similar picture is seen among those patients who tested positive for Entamoeba spp. by rapid triage test. Seasonality has not much effect on the positivity of the patients and infection but it has more to do with the sanitation condition of the household and the surrounding areas $(p<0.001)$.

\section{Conclusion}

Routine screening of intestinal parasites is important to detect silent infections. This will be more beneficial for those having underlying comorbidities or conditions or treatment, which induces immunosuppression.

\section{OrCID}

Nonika Rajkumari @ https://orcid.org/0000-0002-4326-3877

\section{References}

1. Rashid MK, Joshi M, Joshi HS. Prevalence of intestinal parasites among school going children in Bareilly district. Natl J Integr Res Med 2011;2(1):35-37. DOI: http://nicpd.ac.in/ojs-/index.php/njirm/ article/view/1894.

2. Vesy CJ, Peterson WL. The management of Giardiasis. Aliment Pharmacol Ther 1999;13(7):843-850. DOI: 10.1046/j.1365-2036. 1999.00537.x.

3. Choy SH, Al-Mekhlafi HM, Mahdy MA, et al. Prevalence and associated risk factors of Giardia infection among indigenous communities in rural Malaysia. Sci Rep 2014;4:6909. DOI: 10.1038/srep06909.

4. Adam RD. Biology of Giardia lamblia. Clin Microbiol Rev 2001;14(3): 447-475. DOI: 10.1128/CMR.

5. Jayalakshmi S, Dharanidevi S. The Prevalence of Intestinal Parasitic Infections in a tertiary care hospital in southern India-a retrospective study. Int J Curr Microbiol App Sci 2016;5(10):718-723. DOI: $10.20546 /$ ijcmas.2016.510.078.

6. Laishram S, Kang G, Ajjampur SS. Giardiasis: a review on assemblage distribution and epidemiology in India. Indian J Gastroenterol 2012;31(1):3-12. DOI: 10.1007/s12664-012-0161-9.

7. Waldram A, Vivancos R, Hartley C, et al. Prevalence of Giardia infection in households of Giardia cases and risk factors for household transmission. BMC Infect Dis 2017;17(1):486. DOI: 10.1186/s12879-017-2586-3.

8. Reynoso-Robles R, Ponce-Macotela M, Rosas-López LE, et al. The invasive potential of Giardia intestinalis in an in vivo model. Sci Rep 2015;5:15168. DOI: 10.1038/srep15168.

9. Wolfe MS. Giardiasis. Clin Microbiol Rev 1992;5(1):93-100. DOI: 10.1128/ cmr.5.1.93. 
10. Beer KD, Collier SA, Du F, et al. Giardiasis diagnosis and treatment practices among commercially insured persons in the United States. Clin Infect Dis 2017;64(9):1244-1250. DOI: 10.1093/cid/cix138.

11. Khairnar K, Parija SC. A novel nested multiplex polymerase chain reaction (PCR) assay for differential detection of Entamoeba histolytica, E. moshkovskii and E. dispar DNA in stool samples. BMC Microbiol 2007;7:47. DOI: 10.1186/1471-2180-7-47.

12. Hooshyar H, Rostamkhani P, Arbabi M, et al. Giardia lamblia infection: review of current diagnostic strategies. Gastroenterol Hepatol Bed Bench 2019;12(1):3-12. DOI: https://www.ncbi.nlm.nih.gov/pmc/ articles/PMC6441489/. PMCID: PMC6441489.

13. Kamath KR, Murugasu R. A comparative study of four methods for detecting Giardia lamblia in children with diarrheal disease and malabsorption. Gastroenterology 1974;66(1):16-21. DOI: 10.1016/ S0016-5085(74)80074-0.

14. Lanata CF, Fischer-Walker CL, Olascoaga AC, et al. Global causes of diarrheal disease mortality in children $<5$ years of age: a systematic review. PLoS ONE 2013;8: e72788. DOI: 10.1371/journal.pone.0072788.

15. Litleskare S, Rortveit G, Eide GE, et al. Prevalence of irritable bowel syndrome and chronic fatigue 10 years after giardia infection. Clin Gastroenterol Hepatol 2018;16:1064-1072. DOI: 10.1016/j. cgh.2018.01.022.

16. Feng $\mathrm{Y}$, Xiao L. Zoonotic potential and molecular epidemiology of Giardia species and giardiasis. Clin Microbiol Rev 2011; 24:110-40. DOI: 10.1128/CMR.00033-10.
17. Nkrumah B, Nguah SB. Giardia lamblia: a major parasitic cause of childhood diarrhoea in patients attending a district hospital in Ghana. Parasit Vectors 2011;4(1):163. DOI: 10.1186/1756-33054-163.

18. Fantinatti M, Bello AR, Fernandes O, et al. Identification of Giardia lamblia assemblage $\mathrm{E}$ in humans points to a new anthropozoonotic cycle. J Infect Dis 2016;214(8):1256-1259. DOI: 10.1093/infdis/ jiw361.

19. Quihui-Cota L, Morales-Figueroa GG, Javalera-Duarte A, et al. Prevalence and associated risk factors for Giardia and Cryptosporidium infections among children of northwest Mexico: a cross-sectional study. BMC Public Health 2017;17(1):852. DOI: 10.1186/s12889-0174822-6.

20. Mukherjee AK, Chowdhury P, Rajendran K, et al. Association between Giardia duodenalis and coinfection with other diarrheacausing pathogens in India. Biomed Res Int 2014;2014:786480. DOI: 10.1155/2014/786480.

21. Saurabh K, Nag VL, Dash S, et al. Spectrum of parasitic infections in patients with diarrhoea attending a Tertiary Care Hospital in western Rajasthan, India. J Clin Diagn Res 2017;11(8):DC01-DC04. DOI: 10.7860/ JCDR/2017/29001.10346.

22. Sitatow B, Mekuriaw H, Damtie D. Prevalence of intestinal parasitic infections and associated risk factors among Jawi primary school children, Jawi town, north-west Ethiopia. BMC Infect Dis 2019;19(1):341. DOI: 10.1186/s12879-019-3971-x. 